\title{
106 - Environmental Influences on the Cognitive and Psychological Well Being of Older Adults with Dementia
}

\author{
Chair: William E. Reichman \\ Presenters: L. Bradford Perkins \\ Hilde Verbeek
}

This symposium will review the latest data on the influence of environmental design and its attributes on the cognitive and psychological wellbeing of older adults living with dementia. The presenters will cover the myriad ways in which the physical environment of care can adapt to the changing demands of older adults with sensory, motor and cognitive deficits and foster optimal functioning and quality of life. The role of emerging technologies will also be reviewed as they complement the contribution of the design of the physical environment to the wellbeing of older adults with cognitive impairment. Information will be offered through a review of the existing research literature as well as case studies that illustrate the impact of environmental modification on fostering wellbeing and minimizing the emergence of the behavioral and psychological symptoms of dementia. The presenters will represent and integrate sensibilities that have emerged from the fields of architecture, cognitive neuroscience and psychology.

\section{How the Principles of the Culture Change Movement Inform Environmental Design and the Application of Technology in the Care of Older Adults Living with Dementia} William E. Reichman

The culture change movement informs a number of principles that have been applied to more contemporary design concepts for the congregate care of older adults living with dementia. This talk will review the core tenets of the Culture Change Movement as exemplified by the Greenhouse, Dementia Village and other innovative models of congregate long-term care. Specific reference will be made to how these tenets have been operationalized around the world into the design of programming and the creation of residential care environments that foster a better quality of life for older adults and an enhanced work environment for care providers. This talk will also include the emerging role of technologies that complement innovative design of the environment and which foster optimized social and recreational functioning of older adults living with dementia.

\section{A Better Life Through a Better Nursing Home Design}

\section{Bradford Perkins}

Over the last 20 years there has been extensive experimentation related to the role of the environment in the housing, care and treatment of persons with Alzheimer's and other age related dementias. Prior to that time the typical housing and care environment was a locked unit in a skilled nursing or other restrictive senior living facility. In 1991 the Presbyterian Association on Aging in Western Pennsylvania opened Woodside Place on its Oakmont campus. This small 36 bed facility was designed to incorporate the latest research and care experience with persons suffering from these issues. This one small project, as well as the long post occupancy research led by Carnegie Mellon University, clearly demonstrated that individuals with Alzheimer's and related forms of dementia could lead a healthier, happier, higher quality of life in a more residential, less restrictive environment. Not everything in this pioneering project worked, and five generations of living and care models have followed that have refined the ideas first demonstrated by Woodside Place. Bradford Perkins, whose firm designed Woodside Place and over 100 other related projects, will discuss what was learned from Woodside Place as well as the five generations of projects (and post occupancy research) that followed. 
Innovative dementia care environments as alternatives for traditional nursing homes: evidence and experiences from the Netherlands

Hilde Verbeek

Key goals of the dementia care environment focus on increasing autonomy, supporting independence and trying to enable one's own lifestyle for as long as possible. To meet these goals, innovative, smallscale and homelike care environments have been developed that have radically changed the physical, social and organizational aspects of long-term care in the Netherlands. This presentation discusses various Dutch models that have implemented small-scale and homelike care environments, including green care farms, dementia village and citizen initiatives. The models reflect a common care concept, focusing on residents' remaining strengths, providing opportunity for choice and aiming to sustain a sense of self and control. A small number of residents (usually 6 to 8 ) live together in a homelike environment and nursing staff are part of the household. Residents are encouraged to participate in daily household activities, emphasizing normalization of daily life with person-centred care. The physical environment resembles an archetypal home. This talk presents the scientific evidence on the impact and effects of these small-scale, homelike models on residents, their family caregivers and staff. Furthermore, the presentation will highlight working approaches and how these initiatives have positively influenced routine care across the long-term care spectrum. 